\title{
Ausstellung "1914-1918. In Papiergewittern. Die Kriegssammlungen der Bibliotheken" in der Württembergischen Landesbibliothek
}

\author{
von Hans-Christian Pust
}

Am 12. März wurde die Ausstellung "1914-1918. In Papiergewittern. Die Kriegssammlungen der Bibliotheken" in der WLB eröffnet. Die Ausstellung ist ein Gemeinschaftsprojekt von vier Bibliotheken, neben der Bibliothek für Zeitgeschichte in der WLB haben drei französische Bibliotheken mitgearbeitet: Die Partnerbibliothek der WLB, die Bibliothèque nationale et universitaire Strasbourg, die Bibliothèque de documentation internationale contemporaine (Paris) und die Bibliothèque nationale de France (Paris).

In mehrjähriger Vorbereitungszeit haben die vier Bibliotheken eine gemeinsame Ausstellung konzipiert, die das Phänomen der Kriegssammlungen beleuchtet. Schon im Ersten Weltkrieg begannen sowohl zahlreiche öffentliche Stellen wie auch Privatleute, eigene Sammlungen anzulegen, die sich mit der aktuellen Zeit des Weltkriegs beschäftigten. Für 1917 sind in einem Verzeichnis alleine auf deutschem Gebiet über 200 solcher Sammlungen bekannt. In Deutschland gründete sich sogar ein eigener "Verband deutscher Kriegssammlungen", der auch eine eigene Zeitschrift herausbrachte.

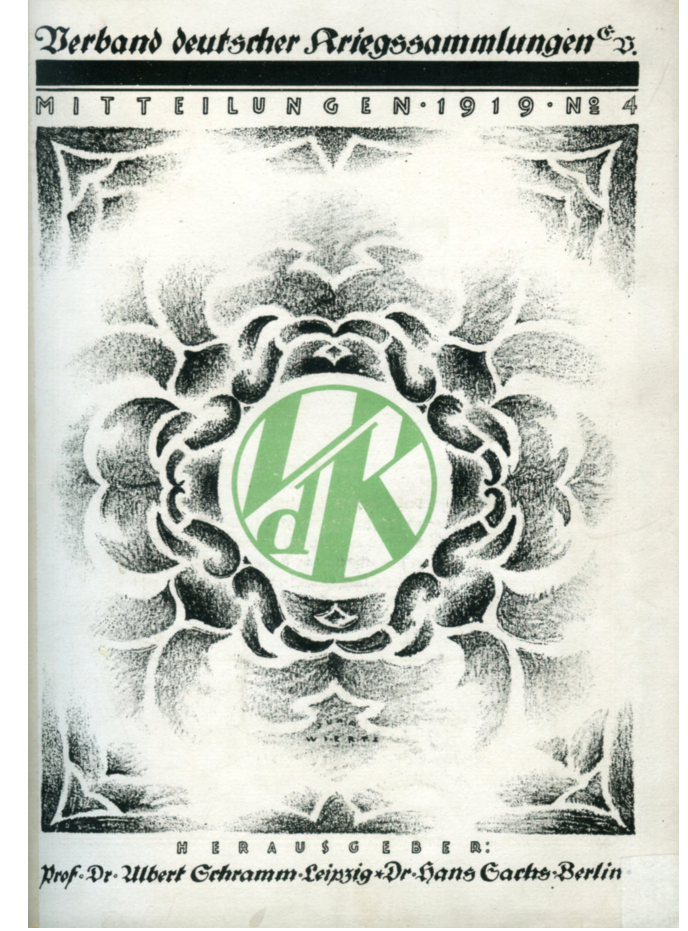

Verband deutscher Kriegssammlungen e.V.: Mitteilungen, 1919, Nr. 4. [Privatsammlung] 
Zu diesen Sammlungen zählte auch die "Weltkriegsbücherei", die 1915 vom schwäbischen Industriellen Richard Franck in Berlin gegründet wurde. Franck hatte alleine 24 Mitarbeiter für diese Aufgabe abgestellt und sammelte über seine zahlreichen Firmenvertreter Material aus dem In- und Ausland. In einem Aufruf an seine Mitarbeiter nannte Franck als besondere Sammlungsbestandteile: Kriegsliteratur, Zeitungen und Zeitschriften, Kriegschroniken, ausländische Blätter und Zeitschriften, Kriegsbroschüren, politische Veröffentlichungen, politische Aktenbücher, Feldpostbriefsammlungen, Kriegsbilderwerke, Ansichten und Fotografien von den Kriegsschauplätzen, Plakate, Schützengrabenzeitungen, Soldatenkunst, Kriegsandenken, Fliegerzettel, Maueranschläge u.a. Die Bibliothek bestand auch nach dem Krieg fort, sie zog 1921 nach Stuttgart um und war bis zur Zerstörung 1944 im Schloss Rosenstein untergebracht. Nach dem Zweiten Weltkrieg kamen die Reste der Bibliothek in das Gebäude der Württembergischen Landesbibliothek.

Eine ganz ähnliche Sammlung stellt die private Sammlung des französischen Industriellenehepaares Louise und Henri Leblanc dar, die schon 1914 ins Leben gerufen wurde. Diese Sammlung sollte sämtliche Relikte des Krieges sammeln, sowohl klassisches Bibliotheksmaterial als auch museale Gegenstände. Bis 1916 waren schon über 22.000 Objekte zusammengetragen worden. Die Sammlung wurde 1917 vom französischen Staat übernommen. Heute wird die Sammlung als Bibliothèque de documentation internationale contemporaine fortgeführt.

Neben diesen beiden privaten Initiativen ist die Kriegssammlung an der Straßburger Kaiserlichen Universitäts- und Landesbibliothek, wie sie bis 1918 hieß, ein Beispiel für eine Kriegssammlung in einer schon bestehenden öffentlichen Einrichtung. Über diese Sammlung ist heute nicht mehr viel bekannt. Teile der Sammlung wurden 2003 bei den Arbeiten zur Ausstellung "Impression d'Europe" wiederentdeckt - der Ausgangspunkt für die Idee einer gemeinsamen Ausstellung zu diesem Thema.

Wie andere Nationalbibliotheken so begann auch die Bibliothèque nationale de France $(\mathrm{BnF})$ in Paris im Ersten Weltkrieg mit dem Aufbau einer Kriegssammlung. Besonders interessant ist dabei der Bereich der Feldzeitungen, da die BnF nach einem Aufruf zahlreiche französische Feldzeitungen erhielt, teilweise sogar mit ausführlichen Begleitschreiben. In der Stuttgarter Ausstellung können die Objekte aus der BnF leider nur als Reproduktionen gezeigt werden.

Neben einigen Exponaten zu den Kriegssammlungen allgemein und den vier Einrichtungen im einzelnen zeigt die Ausstellung überwiegend Exponate, die aus den Kriegssammlungen selbst stammen, gegliedert nach den verschiedenen Medienarten.

Zu einem Großteil bestanden die Sammlungen aus Büchern. Dieser Tatsache wird in der Stuttgarter Ausstellung dadurch Rechnung getragen, dass eine Vielzahl von Büchern aus dem Original-Bestand der damaligen "Weltkriegsbücherei" gezeigt wird. Man sieht, dass der Krieg eine unübersehbare Menge an Buchveröffentlichungen zu allen Lebensbereichen hervorbrachte: Kriegskinderbücher, Kriegsschulbücher, Kriegskochbücher, Reiseführer und Wörterbücher für Soldaten, medizinische Dissertationen über Kriegsverletzungen uvm. 


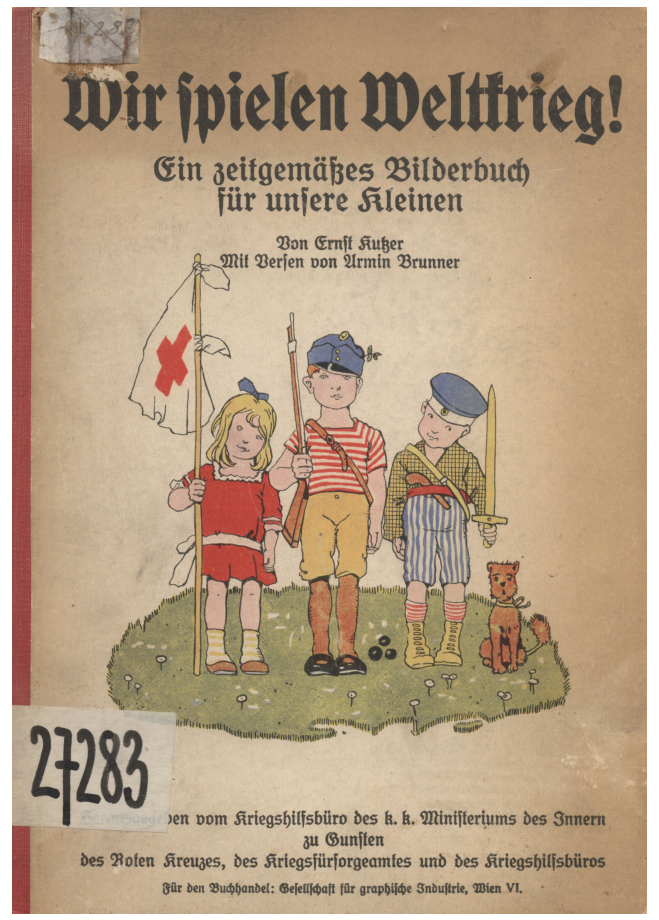

Ernst Kutzer u.a.: Wir spielen Weltkrieg!

Ein zeitgemäßes Bilderbuch für unsere Kleinen, Wien [1915] [BfZ]

Eine weitere wichtige Mediengattung in der Zeit des Ersten Weltkriegs stellten die Postkarten dar - in einer Zeit ohne Radio und Fernsehen eines der Massenkommunikationsmittel der Zeit. In zwei Vitrinen sind 17 deutsche Postkarten aus den Beständen der BfZ und 15 französische Postkarten aus den Beständen der BDIC zu sehen.

Ein relativ neues Medium zu Kriegsbeginn war die Fotografie; in der Stuttgarter Ausstellung stehen deutschen Fotos und Fotoalben aus den Beständen der BfZ und aus Privatbesitz französische Fotos und Fotoalben aus den Beständen der BDIC gegenüber.

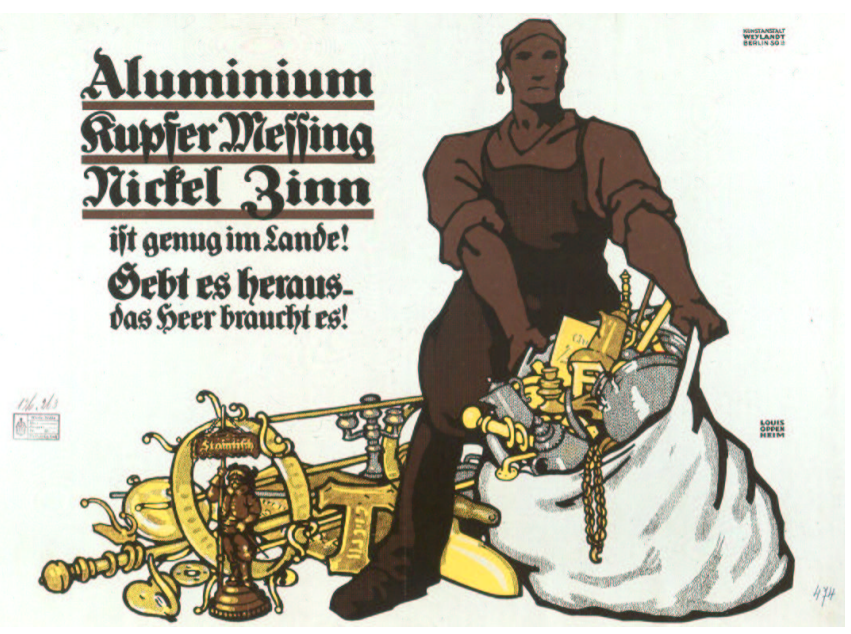

Louis Oppenheim: Aluminium Kupfer Messing Nickel Zinn ist genug im Lande! Gebt es heraus, das Heer braucht es!, Plakat, Lithographie, Berlin 1917. [BfZ] 
Im Ersten Weltkrieg produzierte das Deutsche Reich erstmals eigene Propagandaplakate, teilweise wurden sie von bedeutenden Grafikern wie Fritz Erler oder Ludwig Hohlwein entworfen. In der Ausstellung sind sieben Plakate aus den Beständen der BfZ zu sehen. Die sieben französischen Pendants aus den Beständen der BnF sind leider nur in Reproduktionen zu sehen.

Von den Plakaten zu unterscheiden sind sog. "Maueranschläge", die nur aus Text bestehen und Bekanntmachungen oder Aufrufe enthalten. Um dies zu verdeutlichen sind diese Objekte, von denen zwei aus den Beständen der BfZ und neun aus den Beständen der BNU stammen, auf eine Plexiglaswand aufgezogen. Diese Präsentation soll die ursprüngliche Verwendung dieser Blätter andeuten.

Ein weiteres Medium stellen Flugblätter dar, die sich jeweils an den Kriegsgegner wandten und oft über feindlichem Gebiet abgeworfen wurden. In der Ausstellung sind zahlreiche Exponate aus diesem Bereich zu sehen, die alle aus den Beständen der BNU stammen. Präsentiert werden sowohl alliierte Propaganda-Flugblätter, die sich auf deutsch an die Deutschen wandten wie auch deutsche Flugblätter auf französisch oder pazifistische Flugblätter.

Neben diesen neuen oder doch relativ neuen Medien wurden im Ersten Weltkrieg auch alte Medien reaktiviert. In der Ausstellung stehen dafür beispielhaft Medaillen, Gemälde und Grafiken sowie die sog. "Vivatbänder".

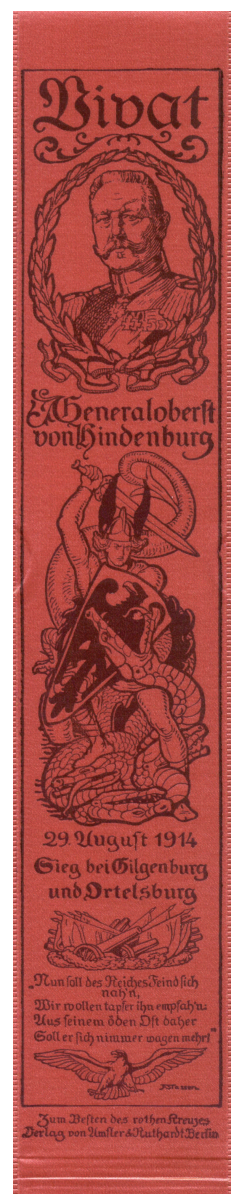

Franz Stassen: Vivatband Vivat Generaloberst von Hindenburg. 29. August 1914. Sieg bei Gilgenburg und Ortelsburg, Berlin: Amsler \& Ruthardt 1914 [BfZ] 
In der Abteilung "Medaillen" stehen sechs französischen Medaillen sechs deutsche Medaillen gegenüber. Alle ausgestellten Medaillen stammen aus den Beständen der BDIC.

Ebenfalls aus den Beständen der BDIC stammen sämtliche Grafiken und Gemälde, die in der Stuttgarter Ausstellung jedoch ebenfalls nur als Reproduktionen gezeigt werden können.

Ein besonderes Kuriosum aus den Beständen der Bibliothek für Zeitgeschichte bieten daneben die Vivatbänder: Seidenbänder mit Text- und Bildaufdruck, die aus Anlass von militärischen Siegen produziert und zu Spendenzwecken verkauft und zahlreich gesammelt wurden.

Als letzte Mediengruppe, die in der Ausstellung gezeigt wird, sind die Medien von der Front zu nennen. Hierzu zählen sowohl Tagebücher und Briefe als auch die sog. "Feldzeitungen", also Zeitungen, die von Soldaten für Soldaten an den Fronten produziert wurden und die sowohl an den Fronten als auch in der Heimat kursierten. Die in Stuttgart gezeigten überwiegend deutschen Feldzeitungen stammen aus den Beständen der BfZ und der BNU.

Tagebücher und Briefe, die sich ebenfalls zahlreich erhalten haben, sind die persönlichsten Zeugnisse, die in den Kriegssammlungen gesammelt wurden. In der Ausstellung sind französische und deutsche Objekte im Original aus den Beständen der BfZ und der BDIC zu sehen. Die Texte sind jeweils transkribiert.

Die Ausstellung war bis zum 25. April 2009 in Stuttgart zu sehen. An insgesamt acht Terminen wurden öffentliche Führungen angeboten, davon zwei in französischer Sprache. Zur Ausstellung ist ein umfangreicher Katalog in einer deutschen und einer französischen Fassung erschienen. Im Rahmenprogramm zur Ausstellung boten die Bibliothek für Zeitgeschichte und die Württembergische Bibliotheksgesellschaft insgesamt drei Vorträge an. 